\title{
Potential applicability of perioperative thromboelastography to access the coagulopathies in live related renal transplantation: a prospective observational pilot study
}

\author{
Amal Francis Sam ${ }^{1}$, Sandeep Sahu ${ }^{2}$, Karthik Ponnappan ${ }^{1}$ \\ ${ }^{1}$ Department of Anaesthesia, Institute of Liver and Biliary Sciences, New Delhi, India \\ ${ }^{2}$ Department of Anaesthesiology and Critical Care, Sanjay Gandhi Postgraduate Institute of Medical Sciences, Lucknow, India
}

Background: Organ transplantation is associated with ischemic and reperfusion injury. Ischemic reperfusion injury (IRI) during liver transplantation results in coagulopathy caused by the release of heparin like substances and platelet trapping. During renal transplantation IRI may be associated with a similar phenomenon, and thromboelastography (TEG) can be used to detect and manage coagulopathy in renal transplantation surgeries.

Methods: TEG was done on pre-operative, immediate post-reperfusion and post-operative day 1 (POD1), for 25 consecutive cases of live related renal transplantation. Coagulopathy was defined as deranged and abnormal TEG variables and supported by the clinical presence of non- surgical oozing and bleeding in the surgical field.

Results: The post reperfusion TEG values showed coagulopathic changes. The $64 \%$ patients had R-time (RT) more than 12 minutes, $64 \%$ patients showed maximum amplitude (MA) less than $55 \mathrm{~mm}$, and $76 \%$ patients had alpha angle less than $55^{\circ}$. The pre-operative TEG coagulation index $(\mathrm{Cl})$ was $2.45 \pm 1.25$, post-reperfusion $\mathrm{Cl}$ was $-1.96 \pm 4.54$ and POD1 $\mathrm{Cl}$ was $4.02 \pm 1.35$. Univariate analysis revealed anti-thymocyte globulin (ATG) and etiology other than chronic glomerulonephritis, as risk factors for the hypocoagulable $\mathrm{Cl}$ in the post reperfusion phase. Changes in $\mathrm{Cl}$ did not translate into symptomatic non-surgical bleeding in the surgical field $\left(\chi^{2}=0.17 ; P=0.67\right)$.

Conclusions: Ischemic reperfusion injury in renal transplantation is associated with transient self-limiting coagulopathy as detected by TEG. Cl values in POD1 indicate a hypercoagulable or prothrombotic state. Whereas immediate post-reperfusion $\mathrm{Cl}$ values show hypocoagulable state. Magnitude of changes shown by TEG did not translate into requirement of blood product transfusion.

Corresponding author: Amal Francis Sam

E-mail: amalfsam@gmail.com

This is an Open Access article distributed under the terms of the Creative Commons Attribution Non-Commercial License (http://creativecommons.org/licenses/by-nc/4.0/) which permits unrestricted non-commercial use, distribution, and reproduction in any medium, provided the original work is properly cited. 